\title{
Raiders of the Lost Wire
}

Authors:

*Carlos H. Salazar, Gabriela Tirado, Angela Mclnerney, Luis NombelaFranco, Ivan Nuñez-Gil

Hospital Universitario Clínico San Carlos, Universidad Complutense, IdISSC, Madrid, Spain.

${ }^{*}$ Correspondence to chsalazart@gmail.com

Disclosure: $\quad$ The authors have declared no conflicts of interest.

Received: $\quad 22.12 .2018$

Accepted: $\quad 12.02 .2019$

Keywords: Complex intervention procedures, fracture of the guidewire, percutaneous coronary intervention.

Citation: $\quad$ EMJ Cardiol. 2019; https://doi.org/10.33590/emjcardiol/18-00120

\section{Abstract}

Introduction: An angiographic guidewire is a basic, yet essential piece of equipment in the interventional cardiologist's armamentarium. Complications associated with angiographic guidewires can be catastrophic to the safe completion of a coronary procedure. In this article, the authors report a case of angiography guidewire fracture and explore the options for management of this rare but serious complication.

Case presentation: A 77-year-old man with multiple cardiovascular risk factors was admitted with an anterior ST segment elevation myocardial infarction. Diagnostic angiography was performed via right radial access and revealed a subocclusive stenosis of the mid and distal left anterior descending artery (LAD) with thrombolysis in myocardial infarction $1 \mathrm{flow}$ and a $70 \%$ stenosis of the proximal diagonal branch. Primary percutaneous coronary intervention was attempted and a HI-TORQUE Balance Middle Weight Universal II guidewire (Abbott Vascular Inc., Santa Clara, California, USA) was chosen. Guidewire manipulation was difficult because of significant calcification and tortuosity of the LAD. Consequently, the guidewire fractured and became trapped in the mid-LAD. The complication was ultimately resolved by stenting across the fractured guidewire and the patient was not afflicted by any adverse sequelae.

Discussion: This case highlights a rare but potentially serious complication of coronary intervention. Proposed management varies from leaving the fractured wire in situ and stenting across it, to varying techniques for removing the fractured wire. However, no consensus exists as to the best strategy. The authors have therefore performed a review of the current literature and propose an algorithm for the management of this rare complication.

\section{INTRODUCTION}

An angiographic guidewire is a basic, yet key piece of equipment in the interventional cardiologist's armamentarium. The complications associated with angiographic guidewires can be catastrophic to the safe completion of a coronary procedure. In this article, the authors report a case of angiography guidewire fracture and explore the options for management of this rare but serious complication. 


\section{CLINICAL CASE}

A 77-year-old man with a history of diabetes, hypertension, and hyperlipidaemia was admitted to the emergency room with an anterior ST segment elevation myocardial infarction (STEMI). Diagnostic angiography was performed via 6F right radial access and revealed a subocclusive stenosis of the mid and distal left anterior descending artery (LAD) with thrombolysis in myocardial infarction (TIMI) I flow, 70\% stenosis of the proximal diagonal branch, and 60\% stenosis of the proximal posterior descending artery. The authors therefore proceeded to primary percutaneous coronary intervention $(\mathrm{PCl})$ of the culprit artery using a 6 -French $\mathrm{XB}$ 3.0 guide catheter and a 0.014" HI-TORQUE Balance Middle Weight (BMW) Universal II guidewire (Abbott Vascular Inc., Santa Clara, California, USA). A $2.0 \times 12 \mathrm{~mm}$ noncompliant balloon was used for initial predilation; however, passage of the balloon was impeded by both the tortuosity and significant calcification of the LAD. A second 0.014" BMW Universal II guidewire to increase support (a 'buddy wire') was advanced into the distal vessel and a guiding catheter extension was also added. Despite this, a second noncompliant balloon failed to transverse the lesion. At this point, it was decided that a wire with greater support was required and therefore a microcatheter was advanced with the intention of exchanging one of the BMW Universal II wires for an ASAHI Grand Slam wire (ASAHI, Tokyo, Japan). However, while retracting the BMW wire it became trapped in the proximal portion of the lesion. Gentle counter traction failed to free the wire, so an attempt was made to remove both the wire and the microcatheter altogether. This manoeuvre, however, resulted in complete fracture of the wire tip, leaving the floppy portion of the wire free-floating in the proximal LAD (Figure 1).

The first strategy employed to resolve this situation was an attempt to retrieve the wire fragment. Multiple wires were advanced into the vessel, with a plan to entrap the fractured segment, but to no avail. Multiple balloon predilations of the lesion were then performed in an attempt to fracture the plaque and free the wire; however, this technique was also unsuccessful. A decision was then made to deploy a stent across the fractured wire tip, thereby trapping it; however, despite multiple attempts to deploy various stents, the operators were unsuccessful. Poor guide-catheter support from the XB 3.0 via the radial artery significantly hampered the attempts at percutaneous stenting. Despite these unsuccessful attempts,
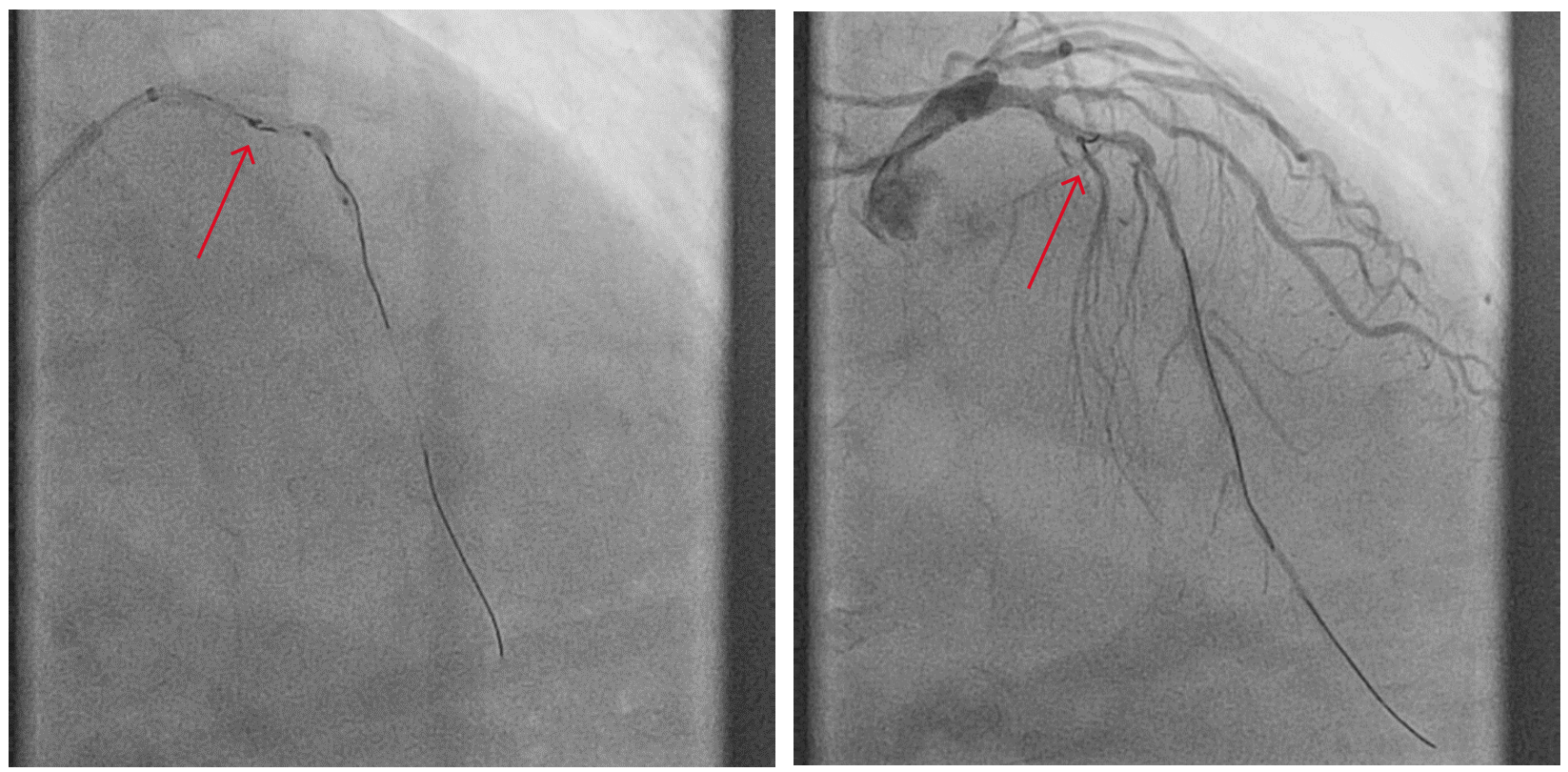

Figure 1: Fractured fragment of guidewire (arrow) in the proximal left anterior descending artery abandoned despite multiple retrieval attempts. 
the culprit vessel now had TIMI 3 flow with resolution of the patient's chest pain and, consequently the procedure was abandoned.

The case was discussed at the authors' multidisciplinary Cardiology-Cardiothoracic Surgery meeting. The consensus agreement was that a second procedure should be undertaken using a different access point with larger sheaths and a larger guide catheter for increased support. The second procedure was performed via femoral access using an 8Fr sheath. The left main stem was engaged with a JL5, 7Fr guide catheter. An ASAHI SION guidewire (ASAHI) was advanced into the distal vessel and exchanged via a microcatheter for an ASAHI Grand Slam wire $(A S A H I)$, which provided the required support. With this extra support, a balloon was passed through the lesion and it was successfully predilated followed by the deployment of three drug-eluting stents covering both the culprit lesion and jailing the free-floating BMW Universal II guidewire tip (Figure 2). The patient recovered without any significant sequelae and remains well at the time of last review.

\section{DISCUSSION}

The fracture or retention of the guidewire is a thankfully rare, but potentially life-threatening, complication that occurs more frequently in complex interventional procedures, such as chronic total occlusion, tortuous vessels, severe calcification, bifurcations, and in-stent restenosis. ${ }^{1-3}$ The estimated incidence is $0.1-0.2 \% .{ }^{1,2}$ Complications can include thrombosis with subsequent vessel occlusion, local or systemic embolisation, vessel perforation with consequent pericardial effusion and tamponade, and wire induced arrhythmias. ${ }^{1-4}$ Prevention of guidewire fracturing is multifaceted and starts with careful selection of the guidewire to be used prior to attempted intervention. Lesion characteristics, such as calcification, tortuosity, and chronicity, influence the choice of guidewire with the correct properties to safely treat the lesion. Excessive rotation while trying to navigate a torturous and/or calcified vessel segment is the main cause of guidewire fracture. Lateral stress generated by excessive torque or bending of the wire tip have been highlighted as potential causes of wire tip fracture and, as such, rotational manipulation of the wire should not exceed 180 degrees. ${ }^{1,2}$
Once the wire tip has fractured and is free-floating in the coronary artery, a dilemma ensues as to the best course of action which, in the absence of consensus opinion, has been informed mainly by case reports and small case series. Three options are available: percutaneous techniques, surgical removal, or conservative management. ${ }^{1,2,5,6}$

\section{Percutaneous Management}

Percutaneous removal is the most attractive and by far the most widely reported technique and in the authors' opinion should be the first method employed. A number of percutaneous options are available, including the 'double' or 'triple' wire technique, whereby a number of angioplasty wires are advanced alongside the retained wire segment and rotated to entangle and remove the free-floating segment; deep wedging of the guide catheter or advancement of a microcatheter such that the free-floating wire tip is in the catheter and a balloon can be advanced and inflated at the mouth of the catheter to trap the wire, with the whole system then being removed en mass; retrieval by snare loop; extraction with bioptome; and laser extraction.,2 All of these techniques have been used in case reports with varying success. ${ }^{1,2}$ Different techniques may be required for different situations. Snare loop and bioptome retrieval are only suitable in vessels of relatively large calibre, with bioptome being more useful when the entrapped wire is near the vessel ostium. Upsizing the guide catheter may be required to accommodate the equipment used for the attempted retrieval and alternative vascular access, such as femoral approach, may also be required. The multiple wire technique represents the simplest wire removal technique and may not require a change of guide catheter. ${ }^{1}$ Another percutaneous option, and the authors' chosen option in this case, is permanent jailing or trapping of the wire behind a stent (i.e., wire exclusion). Of the aforementioned options, exclusion of the wire fragment with a stent is by far the most feasible and involves the least amount of manipulation of the coronary artery, albeit with the trade-off of having wire retained in the vessel. Complete coverage eliminates the risk of migration, and subsequent epithelialisation reduces the risk of thrombosis., ${ }^{1,2}$ The necessary duration of dual antiplatelet therapy after wire exclusion by a stent is currently unclear, with 
no available data. Interval intravascular imaging may be useful in providing the treating physician with some guidance on antiplatelet therapy duration. Percutaneous removal techniques are not without accompanying risks of coronary dissection, rupture, or thrombosis, particularly if excessive manipulation within the coronary vessel is required.

\section{Surgical Management}

Surgical wire removal allows for the option of removing the wire while simultaneously proceeding with coronary artery bypass grafting (CABG), particularly in the case of noncompletion of the original percutaneous stenting procedure. This may be an attractive option if the patient has multivessel disease warranting treatment or has other comorbidities that may make CABG more attractive, such as valvular heart disease or diabetes. However, surgical wire removal carries a mortality risk, as well as significant morbidity, and should therefore be a secondary option if percutaneous techniques unsuccessful. A summary of factors to consider when deciding between percutaneous stenting and CABG is given in Table 1.

Table 1: Factors favouring percutaneous coronary intervention versus coronary artery bypass grafting.

\begin{tabular}{|l|l|}
\hline Favouring percutaneous coronary intervention & Favouring coronary artery bypass grafting \\
\hline - Single vessel disease & - Multivessel disease \\
- Low syntax score & - Left main stem disease \\
- Wire unlikely to be retrieved surgically & - High syntax score \\
& $-\quad$ Concomitant valvular heart disease \\
& - Diabetes \\
& - Low surgical risk \\
\hline
\end{tabular}

Table 2: Preventative strategies.

\begin{tabular}{|c|c|}
\hline \multicolumn{2}{|r|}{ Factors to consider } \\
\hline Access: Radial versus femoral & $\begin{array}{l}\text { - Support required } \\
\text { - Calibre sheath required } \\
\text { - Planned guide catheter } \\
\text { - Equipment required to complete procedure }\end{array}$ \\
\hline Guide catheter & $\begin{array}{l}\text { - Access route to be used } \\
\text { - Support required } \\
\text { - Equipment to be used }\end{array}$ \\
\hline Coronary lesion & $\begin{array}{l}\text { - Severity } \\
\text { - Tortuosity } \\
\text { - Calcification } \\
\text { - Chronicity } \\
\text { - Scoring systems } \\
\text { - AHA classification } \\
\text { - JCTO score }\end{array}$ \\
\hline Guidewire & $\begin{array}{l}\text { - Support } \\
\text { - Torque control } \\
\text { - Trackability } \\
\text { - Coatings }\end{array}$ \\
\hline Procedural aspects & - Avoidance of wire excessive rotation \\
\hline
\end{tabular}

AHA: American Heart Association; JCTO: Japanese chronic total occlusion. 


\section{Conservative Management}

Occasionally, conservative management may be appropriate. Wires that have become fractured and entrapped in distal vessel segments or very small coronary branches are unlikely to cause significant clinical consequences should they thrombose. In these cases, a careful weighing of the risk-benefit ratio of wire retrieval must be carried out and, preferably, in the authors' opinion, in the context of a heart team. A summary of these recommendations is given in Table 2 .

Prevention of wire entrapment and fracture is the first step in avoiding this complication. A number of aspects must be taken into account to avoid wire fracture. Firstly, procedural planning is crucial. In the context of an acute STEMI, as in the presented case, there may be little time for procedural planning; however, in the case of planned $\mathrm{PCl}$, procedural aspects should be well thought out. The route of access must be considered in terms of its ability to accommodate all of the equipment that one plans to use and to provide adequate support in terms of the guide catheter choice. Secondly, the guide catheter must be considered, and a catheter chosen that will both provide adequate support and is of appropriate calibre to accommodate all of the required equipment. Thirdly, the lesion itself must be considered and a thorough assessment of the lesion including severity, tortuosity, calcification, and chronicity must all be taken into account. Finally, the choice of guidewire must be carefully considered. Characteristics of the lesion will dictate the ideal guidewire to use in each circumstance and guidewire properties, such as the support provided, torque control, trackability, and coating properties (hydrophilic versus hydrophobic), need to be carefully considered prior to undertaking the $\mathrm{PCl}$. While all interventional cardiologists employ the use of a 'workhorse' wire in the majority of cases, familiarity with other wires and their properties will allow safer completion of the $\mathrm{PCl}$ and management of complications. In the presented case, the use of a SION wire in the second procedure provided better trackability through the tortuous lesion, and exchanging for a Grand Slam wire provided the support required to railroad the balloons and stents across the lesion. Finally, avoidance of excessive rotation of the wire and maintaining wire rotation to $<180$ degrees greatly decreases the risk of wire fracture as it navigates lesions.
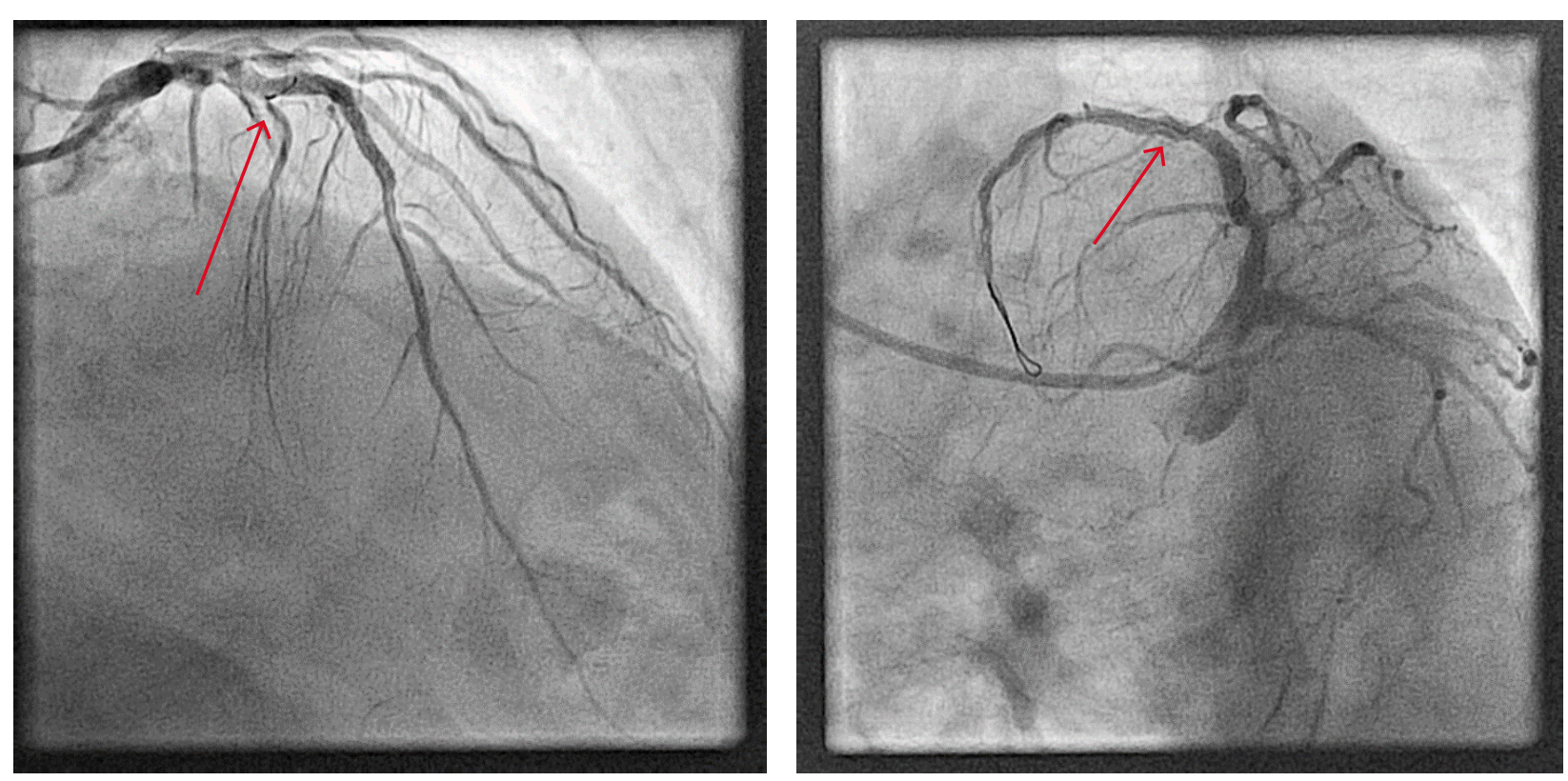

Figure 2: Final result sealing the fragment guidewire (arrow) with a drug-eluting stent with thrombolysis in myocardial infarction III flow. 


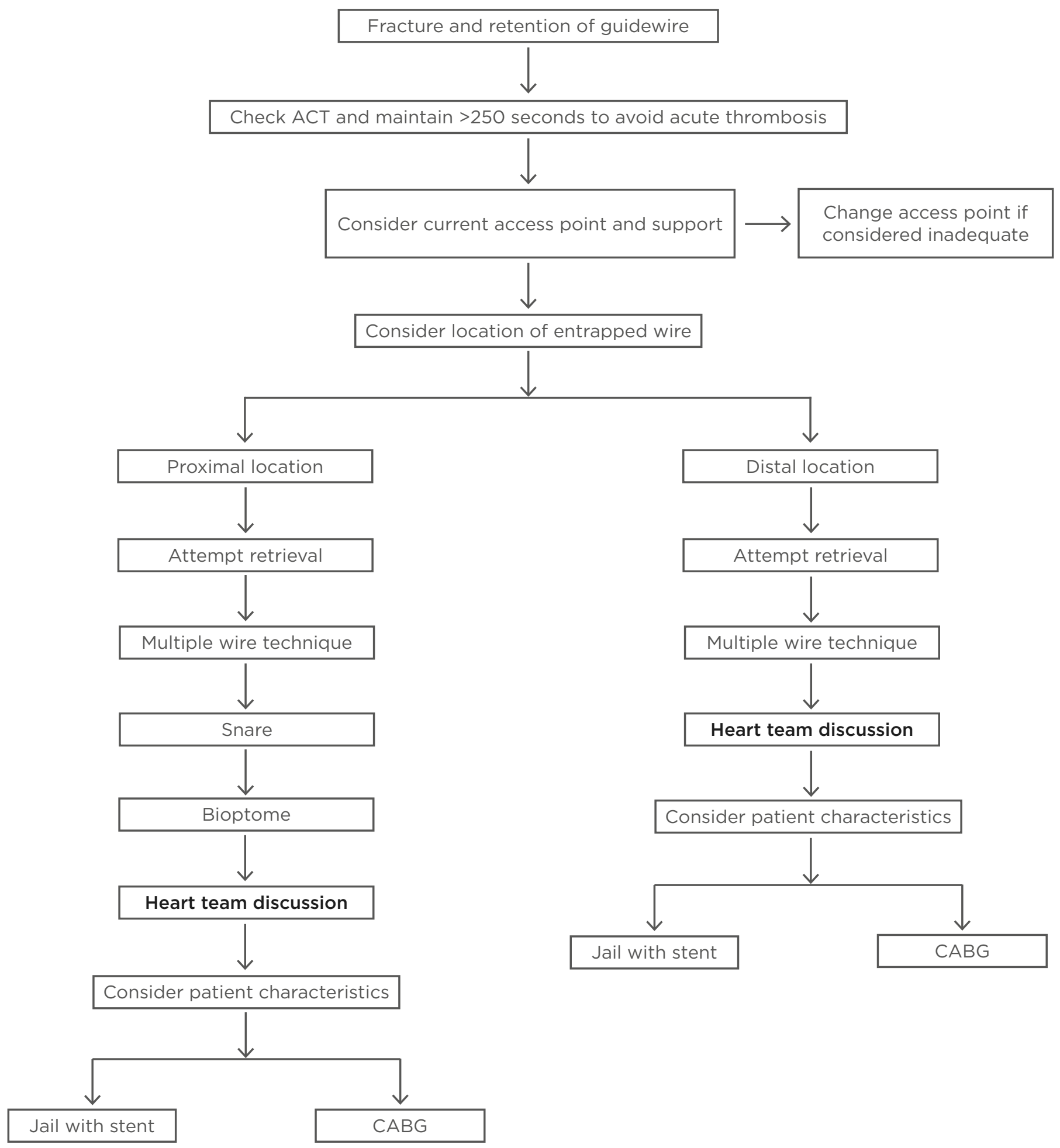

Figure 3: The authors' proposed algorithm for the management of angiography guidewire fracture.

ACT: activated clotting time; CABG: coronary artery bypass grafting.

\section{CONCLUSION}

Based on the above considerations, the authors propose an algorithm for the management of this rare complication, incorporating prevention strategies, as well as a sensible approach to wire retrieval (Figure 3). The authors suggest the proposed management of this complication 
based mainly on the location of the fractured segment, which is the most important factor regarding how to manage this complication. Firstly, because thrombosis can easily occur on the fractured guidewire, the authors recommend that the adequacy of anticoagulation be checked in the first instance by checking activated clotting time and maintaining this for $>250$ seconds with additional doses of unfractionated heparin as required. Secondly, the authors suggest considering the current access point and its ability to provide the required support for what may be a complex procedure. Changing the access point from radial to femoral early in the procedure may save excessive contrast and radiation use, as well as aid in completion of the procedure as efficiently as possible.

Finally, the location of the ruptured wire segment must be considered. If located proximally within the vessel, it is more likely to be amenable to retrieval, whereas distally located ruptured wires may be better managed by jailing behind a stent. Difficult to manage cases should be discussed within the context of the heart team, including interventional cardiologists and cardiothoracic surgeons, and a consensus approach should be taken.

\section{References}

1. Danek BA et al. Consequences and treatment of guidewire entrapment and fracture during percutaneous coronary intervention. Cardiovasc Revasc Med. 2016;17(2):129-33.

2. Al-Moghairi AM, Al-Amri HS. Management of retained intervention guide-wire: A literature review. Curr Cardiol Rev. 2013;9(3):260-6.

3. Kim TJ et al. Fatal subacute stent thrombosis induced by guidewire fracture with retained filaments in the coronary artery. Korean Circ J.
2013;43(11):761-5.

4. Park $\mathrm{SH}$ et al. Retrograde guidewire fracture complicated with pericardial tamponade in chronic total occlusive coronary lesion. Int J Cardiovasc Imaging. 2015;31(7):1293-4.

5. Levine $\mathrm{GN}$ et al. 2011 ACCF/AHA/SCAl guideline for percutaneous coronary intervention a report of the American College of Cardiology Foundation/ American Heart Association Task Force on Practice Guidelines and the Society for cardiovascular angiography and interventions. Circulation. 2011;124(23):2574-609.

6. Windecker $\mathrm{S}$ et al. 2014 ESC/ EACTS Guidelines on myocardial revascularization: The Task Force on Myocardial Revascularization of the European Society of Cardiology (ESC) and the European Association for Cardio-Thoracic Surgery

(EACTS) developed with the special contribution oof the European

Association of Percutaneous

Cardiovascular Interventions (EAPCI). Eur Heart J. 2014;35(37):2541-619. 